\title{
Editorial
}

\section{Image Guided Surgery}

\section{Introduction}

Image-guided systems are essentially like GPS (global positioning satellite) systems for the anatomy of head. These systems are used to aid the surgeon confirming the location of critical structures when the interior of the nose and sinuses is distorted by unusual anatomy or prior surgery. Although first developed for neurosurgery, endoscopic sinus surgery (ESS) rapidly became one of the leading indications for this technology.

In neurosurgery, the primary use of imageguided surgery (IGS) is to locate an intracranial lesion for resection or biopsy. In endoscopic sinus surgery (ESS), the main advantage is to avoid disrupting hazardous areas such as the brain and orbit. The development and rapidly growing popularity of image-guided surgery (IGS) in sinus surgery are directly attributable to the risks of such disruptions

To use the image-guidance navigation system, a CT scan of the sinuses, is performed using a specific navigation system protocol. For some systems, a special mask or markers are placed on the face during the scan to serve as reference points. The CT scan is transferred to a disk, which is then loaded into the image-guidance computer. During surgery, a detection array or a mask is placed on the patient's head. The CT scan images loaded into the system are then calibrated to the patient's anatomy using set pre-set reference points, which may be the mask or markers or specific anatomic points on the face. The position of the sinus surgery instruments can then be tracked by the computer by integrating the information detected from the patient's pre-set reference points and comparing it to the information on the CT scan "map".

\section{History of IGS}

Richard Lebowitz of New York University, School of Medicine, described some of the history behind the development of IGS. The original devices developed about two decades ago required rigid fixation of the head and were trajectory-based.

By the mid-1980s, frameless stereotactic surgery came into play, and had a positionsensitive articulated arm (wands). In the mid1990s IGS progressed to having optical localizers, electromagnetic tracking, and were frameless, allowing for head mobility. At this point, the technology also no longer required use of wands, allowing for greater mobility of instruments.

Then there was a boom in technology, driven by surgeons' needs, Dr. Lebowitz said

"in recent years have seen several advances in endoscopic surgery devices." Now, IGS is used in revision surgery, extended frontal sinus surgery, tumor resection, skull base surgery, endoneurosurgery, and more.

Surgical instruments have become more refined as well. There are straight or curved microdebriders and drills, sinuplasty balloons, and frontal sinus and neurosurgical instruments.

Along with these are advances in CT, MRI, ultrasound, and fluoroscanning, each of which offers ever increasing details. Added to this, there is now the fusing of images, 3D volume rendering, and virtual image updates that are 
continually getting faster. Versions of these imaging devices are smaller and mobile, and can also be used intraoperatively.

IGS instrumentations now include compatible microdebriders-drills, universal instrument adapter systems, and even flexible instruments such as catheters and stents that can be used with hollow core sensors. In addition, ENTs have rotatable curved suction that does not require recalibration as the tip position changes.

There were big changes over the past two decades $\&$ by the next ten years we will be able to see even more and more dramatic changes in IGS. One area to watch for is the development of robotic arms that can be used in conjunction with IGS. In future, it may open the door to things such as remote FESS, where the arm and patient are in one city, and the surgeon in another.

When is image-guidance needed during sinus surgery?

Although the use of image-guidance systems is increasing in endoscopic sinus surgery, it is not required for all sinus procedures. Imageguided surgical navigation is not a substitute for sound surgical judgment and operative experience. Though it is tempting to demand its use during every surgery, it's use does not make much of a difference in sinuses that have not been operated on before. Cases with straightforward anatomy also do not require image-guidance. Furthermore, when they are not indicated, the use of an image-guidance system unnecessarily adds to the length of the procedure and possibly the cost of the procedure.

Image-guidance is most useful while operating on patients who have had previous sinus surgery, patients who have more complicated anatomy of the frontal or sphenoid sinuses, cases of benign tumor growths such as inverted papilloma, and patients who have thinning of the bone between the sinuses and the brain or the eye. If the site of a cerebrospinal fluid (CSF) leak is identified on a preoperative CT scan, the image-guidance system may also be useful during CSF leak repairs to help in isolating the bony defect to be patched. In some cases in which both bony and soft-tissue detail is important, a special technology to merge CT scans, which provide good bony detail, and MRI, which offers better soft tissue detail can be merged to take advantage of both views during the surgery.

\section{Indications of Image Guided Surgery}

The American Academy of Otolaryngology Head and Neck Surgery (AAO-HNS) endorses the use of image-guided surgery for the following procedures:

- Revision sinus surgery

- Distorted sinus anatomy of development, postoperative, or traumatic origin

- Extensive sino-nasal polyposis

- Pathology involving the frontal, posterior ethmoid and sphenoid sinuses

- Disease abutting the skull base, orbit, optic nerve, or carotid artery

- CSF rhinorrhea or conditions where there is a skull base defect

- Benign and malignant sino-nasal neoplasms

\section{Contraindications and Relevant Anatomy}

Computer-assisted endoscopic sinus surgery (ESS) has no absolute contraindications except for lack of experience and training. Physicians must be aware that the technique is an adjunct to surgery and does not replace surgical skills and knowledge.

The surgeon should have through knowledge about the relevant anatomy of the paranasal sinuses, orbits, and base of the skull. 
Advantages of Image-Guided Surgery

- Procedures are less invasive than the open surgical approaches that were once standard.

- Precision is greater, resulting in lower risk to the patient.

- Allows for more complete surgical dissections.

- Difficult sphenoid and ethmoid sinus anatomy can be approached with more surgical confidence.

- Frontal sinus anatomy can be approached with greater confidence, particularly in the presence of false lateral terminal cell.

- Patient's discomfort is minimal with potential for reduced recovery time.

\section{Preoperative Evaluation}

Image-guided surgery (IGS) begins with obtaining a CT scan. The CT scan acquisition protocol used for the authors' needs consists of a helical, 2-mm-thickness axial CT scan with the use of a specially designed headset incorporating built-in metallic fiducial land marking. The specially designed headset allows automatic registration of the imaging to the patient's anatomy in the operating room. The imaging data set is transferred via optical disk, CD-ROM, or computer network to the operating room, where it is loaded into the workstation. The images are brought up on the image-guided surgery (IGS) system prior to the procedure and checked for image quality and accuracy.

\section{Procedural Technique}

Anesthesia is induced on a normal operating table. However, when electromagnetic systems are used, a thick foam mattress is needed to keep the patient off of the metal table in order to prevent interference. With systems using optical technology, the imageguided surgery (IGS) unit must be in direct line to the operating room table with no lineof-sight obstruction, although recent developments alleviated the latter limitation.

\section{Registration}

Registration generates a correlation between the position of the instrument in the surgical field and the corresponding location on the CT images. The instruments are registered to show their position with respect to the orthogonal CT images of the patient. The location is materialized by a set of cross hairs on the screen that moves through the CT image data in concordance with the movement of the pointer.

Image-guided surgery (IGS), using either of the most recent optical or electromagnetic systems, accommodates for head movement. This has enormous implications for surgeons who prefer local or intravenous sedation. Prior to the advent of the new systems, general anesthesia was necessary to ensure absolute fixation of the head relative to the tracking system. With the newer systems, the headset moves along with the head, so registration is maintained throughout the procedure, although frameless registration can also be performed. Therefore, any anesthetic technique may be used.

With an electromagnetic system, the headset that was worn by the patient during the preoperative CT scan acquisition is again applied on the patient's head in the operating room. This correlates head position with the tracking system.

\section{Testing of accuracy}

Accuracy is verified by testing various known landmarks on the patient's face and intranasally to the images on the computer monitor. These locations coordinates are stored and used throughout the procedure to monitor any changes in the accuracy of the device. With most systems, these preliminary steps take less than 2 minutes 
with the collaboration of trained operating room staff. Once registered and verified, the system allows the surgeon to verify surgical position on the monitor depicting the preoperative CT scan in 3 dimensions, along with an additional frame displaying the endoscopic view of the procedure.

\section{Summary \& Conclusion}

Image-guided surgery (IGS) is one of the most significant advances in endoscopic sinus surgery (ESS) since the inception of the endoscopic approach in the mid 1980s. This technology enables the surgeon to follow the anatomical dissection of the sinuses on a computer monitor in the operating room in real time. Difficult anatomic relationships can more easily be understood and treated with the assurance that the critical landmarks are secured. Although the initial expense is substantial, these procedures have minimal per-case costs. IGS should not be considered as a way to palliate lack of experience or understanding of sinonasal surgical anatomy but rather as an adjunctive tool designed for otolaryngologists properly trained in endoscopic sinus surgery (ESS).

Lastly, as the device for IGS is very costly, so it should be used only for the special indications (as mentioned above) in the tertiary level hospital in Bangladesh for giving training to the trainer as well as to the trainee in ENT \& Head-Neck Surgery.

\section{Prof. Khabiruddin Ahmed}

Professor \& Head

Deptt. of ENT \& Head-Neck Surgery

Shaheed Suhrawardy Medical College, Dhaka. 